Proceedings

\title{
Divers Transformations Leading to New Potent GPx Mimetics ${ }^{+}$
}

\author{
Jacek Ścianowski *, Agata J. Pacuła-Miszewska, Magdalena Obieziurska-Fabisiak and Anna \\ Laskowska
}

Department of Organic Chemistry, Faculty of Chemistry, Nicolaus Copernicus University, 7 Gagarin Street, 87-100 Torun, Poland; pacula@umk.pl (A.J.P-M.); magdao@umk.pl (M.O-F.); annlas@doktorant.umk.pl (A.L.)

* Correspondence: jsch@umk.pl

+ Presented at the 1st International Electronic Conference on Catalysis Sciences, 10-30 November 2020; Available online: https://sciforum.net/conference/ECCS2020.

Published: 10 November 2020

\begin{abstract}
Designing a highly active and selective Se-therapeutic, that mimics the activity of the antioxidant enzyme glutathione peroxidase (GPx), still remains a challenge. Since the discovery of ebselen ( $\mathrm{N}$-phenyl-1,2-benzisoselenazol-3(2H)-one) and its ability to act as a GPx mimetic, the search for more effective peroxide scavengers has become a 'hot topic' in this field of research. Herein, we present several modifications of the benzisoselenazolone core that enable to improve the antioxidant and anticancer potential of the basic ebselen structure. These transformations include: (a) installation of chiral terpene skeletons, from $p$-menthane, pinane and carane systems, on the nitrogen atom; (b) exchange of the carbonyl oxygen atom for sulfur to obtain thiocarbonyl derivatives; (c) oxidation of the selenium moiety resulting in a series of benzenoselenenic acids and their further transformation to corresponding water-soluble potassium salts; (d) attachment of an additional phenyl group leading to variously $\mathrm{N}$-substituted unsymmetrical phenylselenides with an $o$-amido function. All of the synthetized compounds were tested as antioxidants and antiproliferative agents. Conclusions concerning the structure-activity correlation, including the difference in reactivity of specific Se-moieties $(-\mathrm{Se}-\mathrm{N}-,-\mathrm{SeOOH},-\mathrm{SeOOK},-\mathrm{SePh}), \mathrm{N}$-substituents (the influence of bulky aliphatic moiety and the 3-dimensional orientation of atoms), and incorporated heteroatoms $(-\mathrm{C}=\mathrm{O},-\mathrm{C}=\mathrm{S})$, are presented.
\end{abstract}

Keywords: ebselen; organoselenium derivatives; antioxidant activity; anticancer activity

\section{Introduction}

Reactive oxygen species (ROS), play an important role as mediators and regulators in the cells physiology [1,2]. However, their intense and long-lasting effect can have a destructive impact on biomolecules, such as DNA, lipids or proteins and consequently cause a whole range of diseases, e.g. cardiovascular disorders, cancer, neurodegeneration and aging [3-5]. Therefore, the production of ROS must be strictly controlled by the enzymatic and non-enzymatic antioxidant systems, in order to avoid the disorder of homeostasis between pro- and antioxidant processes, defined as "oxidative stress" [6,7]. The selenoenzyme-glutathione peroxidase (GPx) is an important part of this biomachinery. Ebselen ( $\mathrm{N}$-phenyl-1,2-benzisoselenazol-3(2H)-one) was one of the first organoselenium compounds to be discovered as a GPx mimic. Although ebselen was found to have very promising antioxidant properties, several side effects and low solubility prompted the search for specific structural modifications that could improve its bioavailable and reduce the observed negative aftereffects. Till now, we have performed several transformations of the benzisoselenazolone core that enable to modify the bio-activity of the basic ebselen structure. These modifications included: a) exchanging the oxygen atom of a carbonyl group for a sulfur atom to form thiocarbonyl derivatives; b) substitution of the nitrogen atom with chiral skeletons; c) oxidation of the Se-N bond to form 
selenenic acids $-\mathrm{SeOOH}$ and its subsequent transformation into the water-soluble seleninic acid potassium salts; d) transformation of the Se-N bond into a selenide moiety (Scheme 1).

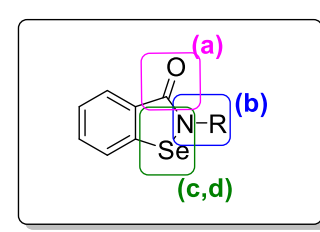
(a) $\mathrm{O} \longrightarrow \stackrel{\mathrm{S}}{\mathrm{N}}$
(b) $\mathrm{R}=$ terpene
(c) $-\mathrm{Se}-\mathrm{N}-\longrightarrow-\mathrm{SeOOH} \longrightarrow-\mathrm{SeOOK}$
(d) $-\mathrm{Se}-\mathrm{N}-\longrightarrow-\mathrm{SePh}$

Scheme 1. Structural modification of $\mathrm{N}$-substituted benzisozelenazol-3(2H)-ones.

The conducted transformations enabled to obtained a variety of Se-based GPx mimics. All derivatives were tested as antioxidants and anticancer agents. The influence of specific modifications on the activity of the molecules is highlighted.

\section{Results and Discussion}

\subsection{Synthesis of Benzisoselenazol-3-(2H)-Thiones}

The exchange of the carbonyl oxygen atom with a sulfur atom, in the structure of benzisoselenazol-3-(2H)-ones, reduces the polarity of the double bond. This can influence the stability of the Se-N bond and its reactivity towards ROS (antioxidant properties) and proteins (the rate of SSe bond formation). Based on this assumption, we have developed an efficient methodology for the preparation of benzisoselenazol-3-(2H)-thions using Lawesson's reagent [8].

$\mathrm{N}$-alkylbenzisoselenazolthiones $\mathbf{3 a}-\mathbf{f}$ were obtained by two different two-step methods. The first of them (Method A) involved the reaction of N-alkyl-o-iodobenzamides 1 with Lawesson's reagent (L.R.), followed by nucleophilic substitution of the obtained thioamides by $\mathrm{Li}_{2} \mathrm{Se} 2$. The second procedure (Method B) was based on the formation of benzisoselenazol-3-(2H)-ones 4 in the reaction of $\mathrm{N}$-alkyl-o-iodobenzamides 1 with $\mathrm{Li}_{2} \mathrm{Se}_{2}$ [9], and then the reaction of ebselen derivatives 4 with Lawesson's reagent (Scheme 2).

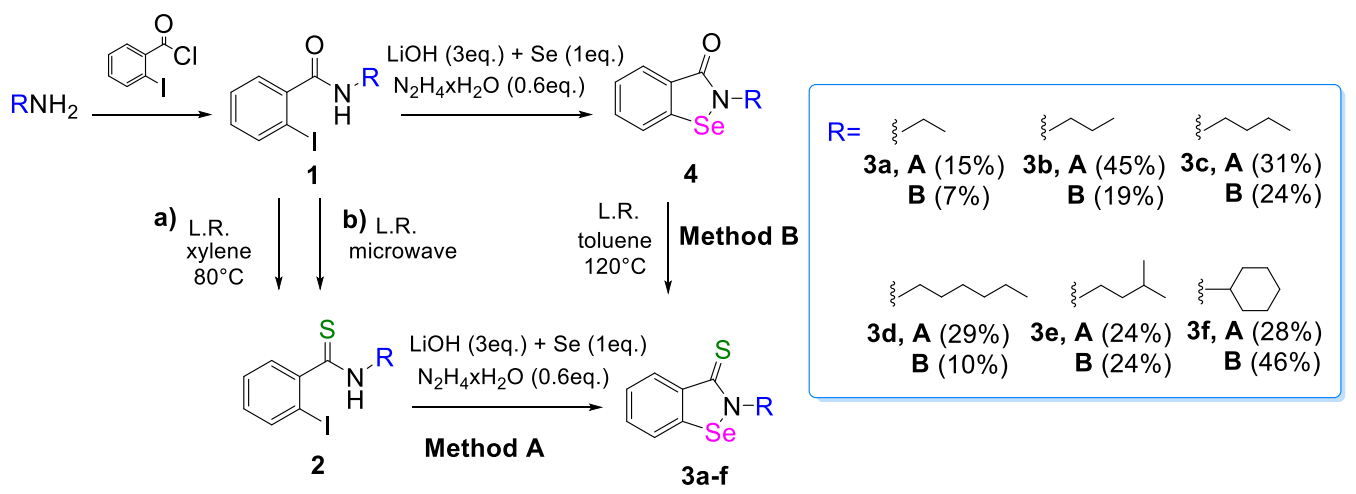

Scheme 2. Methods A and B used to obtain thio-derivatives 3a-f.

The reaction of amides 1 with Lawesson's reagent, carried out under standard conditions (route a) [10], allowed to obtain the thioamides 2 in only moderate yields (reaction time: $12 \mathrm{~h}$, yields: 22 $61 \%$ ). Performing the same reaction using microwave radiation (route b), under solvent-free conditions, significantly shortened the reaction time $(3 \mathrm{~min})$ and improved the yields of the process $(44-82 \%)$.

\subsection{Synthesis of N-Terpenyl Benzisoselenazol-3(2H)-Ones}

As chiral compounds, that possess a strictly defined orientation of substituents on the asymmetric carbon, can interact with specific biological targets differently, depending on their configuration and the structure of the matching receptor, we also wanted to synthetize a series of 
chiral $N$-substituted benzisoselenazolones, including different enantiomers, epimers and regioisomers, and determin the correlation between the structure of the compound and its biological activity.

For this purpose, we have first synthetized a series of terpene amines, by a multistep methodology starting from the corresponding alcohol ( $\mathrm{p}$-menthane system) or alkene (pinene and carene systems), that were further converted to corresponding benzisoselenazol-3(2H)-ones 7-14 by the reaction with 2-(chloroseleno) benzoyl chloride 6 (Scheme 5) [11].

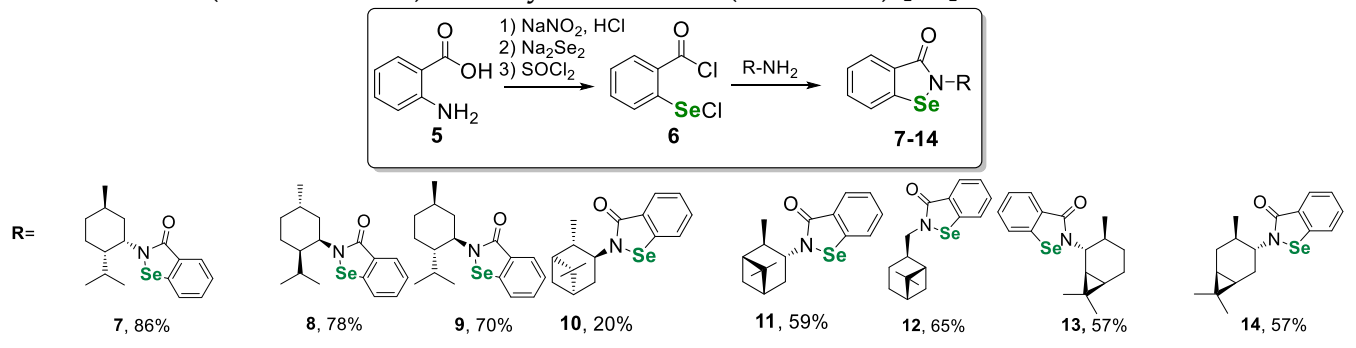

Scheme 3. Synthesis of N-terpenyl benzisoselenazol-3(2H)-ones 7-14.

\subsection{Synthesis of Seleninic Acid Potassium Salts}

The main way to improove the bioavailability of a chemical compound is to increase its solubility in body fluids. Moderate antioxidant activity of ebselen is mainly related to its poor solubility in water, which becomes particularly important when attempting to administer the drug intravenously. To adress this issue, we have conducted the synthesis of water-soluble derivatives in the form of potassium salts of 2-( $N$-alkylcarboxamido)benzeneselenic acids 17a-f. The first step of the research involved the synthesis of $\mathrm{N}$-alkylbenzeneseleneinic acids $16 \mathbf{a}-\mathbf{f}$ with $o$-amide function. The acids $16 a-$ f were obtained using two alternative methods: by oxidation of $\mathrm{N}$-alkylbenzisoselenazol-3(2H)-ones 4a-f (method $\mathrm{C}$ ) or the corresponding diselenides 15a-f (method D) with $30 \% \mathrm{H}_{2} \mathrm{O}_{2}$. Nalkylbenzeneseleninic acids 16a-f in the next step were converted into the corresponding benzeneseleninic salts $\mathbf{1 7} \mathbf{a}-\mathbf{f}$ by reaction with potassium tert-butoxide in anhydrous ethanol (Scheme 4) [12].

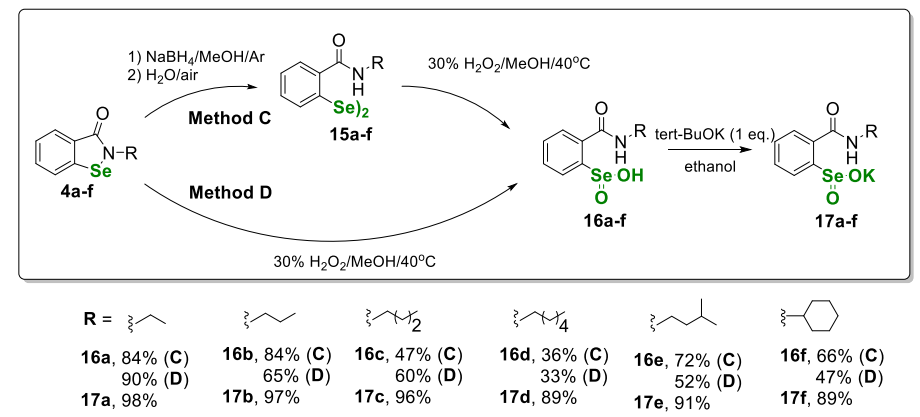

Scheme 4. Sythesis of benzeneseleninic acids $16 a-f$ and corresponding potassium salts $17 a-f$.

\subsection{Synthesis of N-substituted Unsymmetrical Phenylselenides}

The simplicity of including aromatic or heteroaromatic rings in the structure of a compound and the possibility of their easy modification may turn out to be a way to increase the activity of the pharmacophore [13,14]. Taking this into account, we attempted to instal an additional phenyl ring in the structure of ebselen and synthesize a series of $N$-substituted asymmetric phenylselenides bearing an o-amide group $\mathbf{1 9 b}-\mathbf{3 5 b}$.

The first step of the research involved the synthesis of $N$-substituted o-iodobenzamides 19a-35a by the reaction of amines with $o$-iodobenzoic acid chloride 18. The resulting benzamides 19a-35a were converted into the corresponding $N$-aliphatic, $N$-aromatic and chiral $N$-terpene phenylselenides $\mathbf{1 9 b} \mathbf{b} \mathbf{3 5} \mathbf{b}$ using a newly developed procedure involving nucleophilic copper-catalyzed substitution by Senucleophile generated in situ from diphenyl diselenide and sodium borohydride (Scheme 5) [15]. 


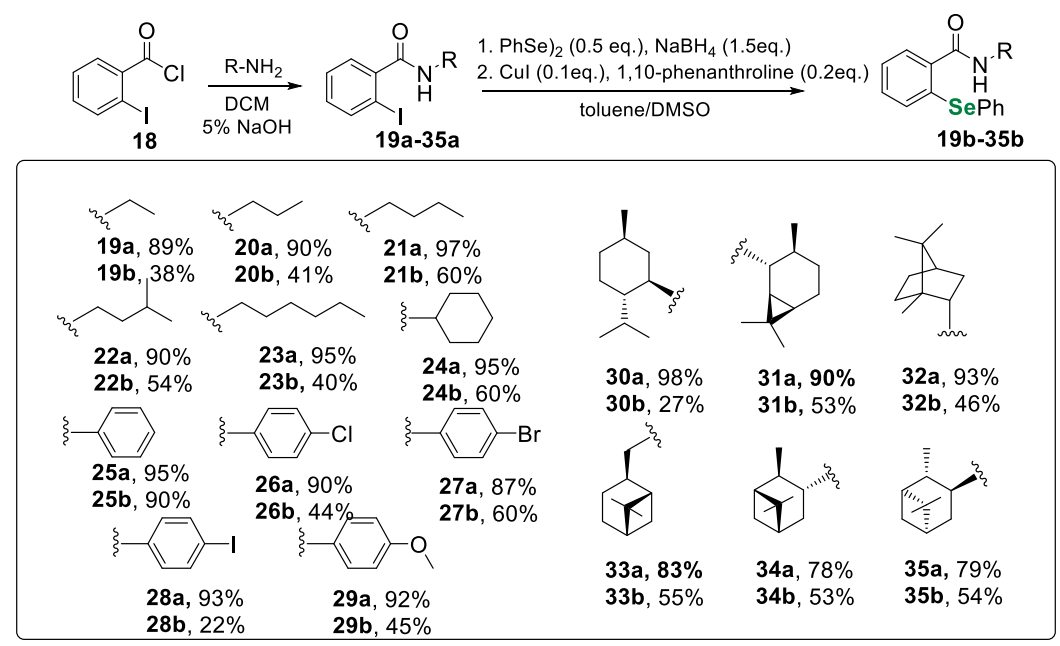

Scheme 5. Synthesis of N-substituted phenylselenides 19b-35b.

\subsection{Evaluation of the Antioxidant Activity}

All obtained derivatives were tested as antioxidants using the popular NMR test developed by Iwaoka and co-workers [16]. The results with the highest antioxidant potential are presented in Table 1.

Table 1. Results of the antioxidant activity measurement.

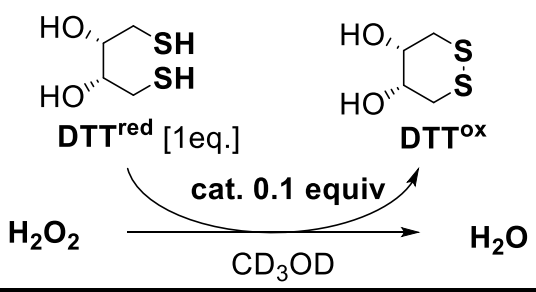

\begin{tabular}{|c|c|c|c|c|c|}
\hline \multirow[b]{2}{*}{ Catalyst [0.1 equiv.] } & \multicolumn{5}{|c|}{ Remaining Dithiotreitol (\%) } \\
\hline & $3 \mathrm{~min}$ & $5 \mathrm{~min}$ & $15 \mathrm{~min}$ & $30 \mathrm{~min}$ & $60 \mathrm{~min}$ \\
\hline \multicolumn{6}{|c|}{ Benzisoselenazolthiones } \\
\hline $3 b$ & 43 & 21 & 3 & 2 & 0 \\
\hline $3 \mathbf{e}$ & 40 & 26 & 18 & 17 & 15 \\
\hline \multicolumn{6}{|c|}{$\mathrm{N}$-terpenyl benzisoselenazol-3(2H)-ones } \\
\hline $10 / 11$ & 71 & 39 & 5 & 0 & 0 \\
\hline 12 & 74 & 61 & 28 & 6 & 0 \\
\hline \multicolumn{6}{|c|}{ Benzeneseleninic Acids } \\
\hline $16 \mathrm{e}$ & 76 & 56 & 38 & 24 & 12 \\
\hline $16 f$ & 85 & 64 & 37 & 18 & 2 \\
\hline \multicolumn{6}{|c|}{ Seleninic Acid Potassium Salts } \\
\hline $17 \mathrm{a}-\mathrm{f}$ & 0 & 0 & 0 & 0 & 0 \\
\hline \multicolumn{6}{|c|}{ Phenylselenides } \\
\hline $21 \mathrm{a}$ & 57 & 39 & 16 & 4 & 0 \\
\hline $22 a$ & 98 & 97 & 94 & 88 & 71 \\
\hline Ebselen & 84 & 75 & 64 & 58 & 52 \\
\hline
\end{tabular}

Due to the fact that the change of the $-\mathrm{SOOH}$ group to the $-\mathrm{SOOK}$ group resulted in a drastic increase in activity (the reaction was completed in $3 \mathrm{~min}$ ) all benzeneseleninic acid salts 17a-f were evaluated by the same procedure but using 0.01 equivalent of the Se-catalyst (Table 2).

Table 2. Results of the antioxidant activity measurement for salts. 


\begin{tabular}{cccccc}
\hline & \multicolumn{5}{c}{ Remaining Dithiotreitol (\%) } \\
\hline Catalyst [0.01 equiv.] & $\mathbf{3}$ min & $\mathbf{5}$ min & $\mathbf{1 5}$ min & $\mathbf{3 0}$ $i n$ & $\mathbf{6 0}$ min \\
\hline & \multicolumn{6}{c}{ Seleninic } & Acid Potassium Salts \\
\hline $\mathbf{1 7 a}$ & 24 & 11 & 0 & 0 & 0 \\
$\mathbf{1 7 e}$ & 59 & 16 & 0 & 0 & 0 \\
Ebselen & 97 & 96 & 95 & 94 & 92 \\
\hline
\end{tabular}

The most important features improving the antioxidant activity was the presence of a bulky substituent, that probably enables the facile cleavage of the Se-N bond ( $N$-terpene derivatives 10-12) and good solubility in water (benzeneseleninic acid salts 17a-f).

\subsection{Evaluation of the Cytotoxic Activity}

The cytotoxic activity of the obtained derivatives was evaluated by the cell viability assay (MTT) on breast cancer MCF-7 [17] and human promyelocytic leukemia HL-60 cell lines. The IC50 values for compunds with the best results are presented in Table 3.

Table 3. Cytotoxic activity evaluated in vitro.

\begin{tabular}{|c|c|c|c|c|c|}
\hline & MCF-7 & HL-60 & & MCF-7 & HL-60 \\
\hline & \multicolumn{2}{|c|}{$\mathrm{IC}_{50,}, \mathrm{MM}$} & \multicolumn{3}{|c|}{$\mathrm{IC}_{50, \mu \mathrm{M}}$} \\
\hline \multicolumn{3}{|c|}{$\mathrm{N}$-terpenyl benzisoselenazol-3(2H)-ones } & \multicolumn{3}{|c|}{ Seleninic acid potassium salts } \\
\hline 10 & $19.9 \pm 0.4$ & $7.1 \pm 0.4$ & $17 f$ & $16.6 \pm 1.1$ & $42.1 \pm 3.1$ \\
\hline 11 & $13.3 \pm 1.1$ & $20.6 \pm 1.0$ & \multicolumn{3}{|c|}{ Phenylselenides } \\
\hline 7 & $12.4 \pm 0.4$ & $12.4 \pm 0.9$ & $31 b$ & $16.35 \pm 0.29$ & $16.3 \pm 0.16$ \\
\hline 8 & $85.5 \pm 4.0$ & $61.3 \pm 3.2$ & \multicolumn{3}{|c|}{ Carboplatin } \\
\hline \multicolumn{3}{|c|}{ Benzeneseleninic acids } & & $0.70 \pm 0.30$ & $3.19 \pm 0.46$ \\
\hline $16 a$ & $40.1 \pm 1.2$ & $11.7 \pm 1.0$ & & & \\
\hline
\end{tabular}

In the case of benzisoselenazolones and phenylselenides the attachment of chiral bulky terpene substituents seemed to enhance the cytotoxic potential. Although, the antiproliferative activity of all derivatives was lower than for the known drug carboplatin, the difference of reactivity of two enantiomeric pairs $N$-pinocampheyl 10 and $\mathbf{1 1}$ and $N$-menthyl derivatives 7 and 8 present an interesting example that the biological activity can be selectively modified by incorporating specific chiral structures on the nitrogen atom of the benzisoselenazolone core.

\section{Conclusions}

Herein, we have presented various modifications of the benzisoselenazolone core that enable to improve the antioxidant and anticancer potential of the basic ebselen structure. The compounds with the highest antioxidant potential were the benzeneseleninic acid potassium salts $17 \mathbf{a}-\mathbf{f}$. The best obtained antioxidant was 2-(N-ethylcarboxyamido)benzeneselenenic acid potassium salt 17 a, used in only 0.01 equivalent, for which the lack of substrate was observed after the $15 \mathrm{~min}$ of reaction time. Among all tested derivatives, the highest antioxidant activity was observed for compounds with a 3methylbutyl substituent. The highest antiproliferative potential towards HL-60 cell line exhibited $\mathrm{N}$ isopinocampheyl-1,2-benzisoselenazol-3(2H)-one 10 (IC50 of $7.1 \pm 0.4 \mu \mathrm{M}$ ) and against MCF7 the $N$ menthyl-1,2-benzisoselenazol-3(2H)-one 7 (IC50 of $12.4 \pm 0.4 \mu \mathrm{M}$ ).

Author Contributions: Conceptualization, J.S.; methodology, J.S.; formal analysis, A.J.P-M., M.O-F. and A. L.; investigation, A.J.P-M., M.O-F. and A.L.; data curation, A.J.P-M. and M.O-F.; writing-original draft preparation, A.J.P-M.; writing-review and editing, J.S. All authors have read and agreed to the published version of the manuscript.

Funding: This research received no external funding.

Conflicts of Interest: The authors declare no conflict of interest. 


\section{References}

1. Schieber, M.; Chandel, N.S. ROS function in redox signaling and oxidative stress. Curr. Biol. 2014, 24, R453-R462.

2. Sies, H. Oxidative stress: a concept in redox biology and medicine. Redox Biol. 2015, 4, 180-183.

3. Davies, K.J. Protein damage and degradation by oxygen radicals. I. general aspects. J. Biol.Chem. 1987, 262, 9895-9901.

4. Terman, A.; Brunk U.T. Oxidative Stress, Accumulation of Biological 'Garbage', and Aging. Antioxid. Redox Signal. 2006, 8, 197-204.

5. Dalle-Donne, I.; Aldini, G.; Carini, M.; Colombo, R.; Rossi, R.; Milzani, A.J. The effect of mesedin on the content of oxidative stress biomarkers in the brain tissue in ischemia. Cell Mol. Med. 2006, 10, 389-406.

6. Finkel T.; Holbrook N. Oxidants, oxidative stress and the biology of ageing. Nature 2000, 408, $239-247$.

7. Sies H. Oxidative stress: a concept in redox biology and medicine. Acad. Press 1985, 4, 1-8.

8. Obieziurska M.; Pacuła A.J.; Juhas U.; Antosiewicz J.; Ścianowski J. The Influence of O/S exchange on the Biocatalytical activity of benzisoselenazol-3(2H)-ones. Catalysts 2018, 8, 493-507.

9. Pacuła, A.J.; Ścianowski, J.; Aleksandrzak, K.B. Highly efficient synthesis and antioxidant capacity of Nsubstituted benzisoselenazol-3(2H)-ones. RSC Adv. 2014, 4, 48959-48962.

10. Scheibye, S.; Kristensen, J.; Lawesson, S.O. Studies on organophosphorus compounds - XXVII1: Synthesis of thiono-, thiolo- and dithiolactones. Tetrahedron 1979, 35, 1339-1343.

11. Obieziurska M.; Pacuła A.J.; Długosz-Pokorska A.; Krzemiński M.; Janecka A.; Ścianowski J., Bioselectivity induced by chirality of new terpenyl organoselenium compounds. Materials 2019, 12, 3579-3591.

12. Obieziurska M.; Pacuła A.J.; Laskowska A.; Długosz-Pokorska A.; Janecka A.; Ścianowski J., Seleninic acid potassium salts as water-soluble biocatalysts with enhanced bioavailability. Materials 2020, 3, 661 .

13. Polêto, M.D.; Rusu, V.H.; Grisci, B.I.; Dorn, M.; Lins, R.D.; Verli, H. Aromatic rings commonly used in medicinal chemistry: Force fields comparison and interactions with water toward the design of new chemical entities. Front. Pharmacol. 2018, 9, 395-414.

14. Ward, S.E.; Beswick, P. What does the aromatic ring number mean for drug design? Expert Opin. Drug Discov. 2014, 9, 9-18.

15. Obieziurska-Fabisiak M.; Pacuła A.J.; Capoccia L.; Drogosz-Stachowicz J.; Janecka A.; Santi C.; Ścianowski J. Phenylselanyl group incorporation for "glutathione peroxidase-lLike" activity modulation. Molecules 2020, 25, 3354.

16. Kumakura, F.; Mishra, B.; Priyadarsini, K.I.; Iwaoka, M. A water-soluble cyclic selenide with enhanced glutathione peroxidase-like catalytic activities. J. Org. Chem. 2010, 3, 440-444.

17. Mosmann, T. Rapid colorimetric assay for cellular growth and survival: application to proliferation and cytotoxicity assays. J. Immunol. Methods 1983, 65, 55-63.

Publisher's Note: MDPI stays neutral with regard to jurisdictional claims in published maps and institutional affiliations.

(C) 2020 by the authors. Submitted for possible open access publication under the terms and conditions of the Creative Commons Attribution (CC BY) license (http://creativecommons.org/licenses/by/4.0/). 